\title{
Making ultracold molecules in a two color pump-dump photoassociation scheme using chirped pulses
}

\author{
Christiane P. Koch, ${ }^{1,2}$, 円 Eliane Luc-Koenig, ${ }^{1}$ and Françoise Masnou-Seeuws ${ }^{1}$ \\ ${ }^{1}$ Laboratoire Aimé Cotton, CNRS, Bât. 505, Campus d'Orsay, 91405 Orsay Cedex, France \\ ${ }^{2}$ Department of Physical Chemistry and The Fritz Haber Research Center, The Hebrew University, Jerusalem 91904, Israel
}

(Dated: April 21, 2022)

\begin{abstract}
This theoretical paper investigates the formation of ground state molecules from ultracold cesium atoms in a two-color scheme. Following previous work on photoassociation with chirped picosecond pulses [Luc-Koenig et al., Phys. Rev. A 70, 033414 (2004)], we investigate stabilization by a second (dump) pulse. By appropriately choosing the dump pulse parameters and time delay with respect to the photoassociation pulse, we show that a large number of deeply bound molecules are created in the ground triplet state. We discuss (i) broad-bandwidth dump pulses which maximize the probability to form molecules while creating a broad vibrational distribution as well as (ii) narrow-bandwidth pulses populating a single vibrational ground state level, bound by $113 \mathrm{~cm}^{-1}$. The use of chirped pulses makes the two-color scheme robust, simple and efficient.

PACS numbers: 33.80.Ps,33.80.-b,32.80.Qk,33.90.+h
\end{abstract}

\section{INTRODUCTION}

The field of cold molecules is rapidly developing, since many research groups are working at the production of dense samples of cold $(T \leq 1 \mathrm{~K})$ or ultracold $(T \leq 100$ $\mu \mathrm{K})$ molecules [1]. Moreover, the field of molecular condensates has opened recently, with experimental evidence for molecules formed in a degenerate gas, starting either from an atomic Bose-Einstein condensate 2, 3, 4, 5, 6], or from an atomic Fermi gas [7, 8, 9, 10, 11, 12, 13]. This intense activity is motivated by possible applications to very precise measurements (lifetimes, checks on parity violation, determination of a possible dipole moment of the electron as a check for the standard model in elementary particles), Bose-Einstein condensation of complex systems (molecule laser), or the emergence of a new ultracold chemistry.

Among the various routes to produce ultracold molecules, photoassociation has emerged as a purely optical technique starting from an assembly of laser-cooled atoms in a trap. The reaction takes place when a pair of ground state atoms interacting via the potential $V_{\text {ground }}$ absorbs a photon red-detuned compared to the atomic line. At resonance, one vibrational level in an excited electronic potential $V_{\text {exc }}$ of the molecule is populated. The reaction can be interpreted as a vertical transition at large distances, forming a long range molecule. We shall consider the example of two cold cesium atoms colliding in the lower triplet $a^{3} \Sigma_{u}^{+}(6 s+6 s)$ potential and forming a molecule in a vibrational level $v^{\prime}$ of the $0_{g}^{-}\left(6 s+6 p_{3 / 2}\right)$ potential. The first experimental observation of ultracold $\mathrm{Cs}_{2}$ molecules has made use of this reaction [14].

In order to make molecules in the ground or lower triplet state, the photoassociation step must be followed

*Electronic address: ckoch@fh.huji.ac.il by a stabilization step. The photoassociated molecules are short-lived and decay via spontaneous emission, most often through a vertical transition at large interatomic distances $R$ giving back a pair of atoms. However, specific mechanisms which favor radiative decay at shorter distances exist and allow for populating bound vibrational levels in the potential $V_{\text {ground }}(R)$ (or possibly, for instance in case of heteronuclear dimers, to another potential correlated to the same asymptote 15]). In the special case of the double well $0_{g}^{-}\left(6 s+6 p_{3 / 2}\right)$ excited potential of $\mathrm{Cs}_{2}$, the smooth barrier between the two wells acts as a "speed bump" and slows down the vibrational motion in the external well such that vertical transitions can take place at intermediate distances $R_{\text {int }}$. Stable molecules formed by spontaneous emission through this mechanism have been detected in a MOT [14]. Other efficient stabilization mechanisms such as resonant coupling [16, 17, 18] exist.

In a condensate, in order to retain coherence, spontaneous emission should be avoided and a stabilization step involving stimulated emission should be implemented within a two-color experiment. However, with continuous wave $(\mathrm{cw})$ lasers such experiments are hardly feasible since the scheme is fully reversible: Population is pumped back from bound levels of the ground state to the excited state, and then to continuum levels of the ground state, i.e. the molecule is dissociated. The use of laser pulses becomes thus unavoidable.

In previous work 19, 20, 21], the possibility to increase the efficiency of the photoassociation reaction by use of chirped laser pulses was considered. The frequency of such pulses has a linear time-dependence. The parameters of the pulse were optimized to achieve a total transfer of population under adiabatic following conditions 22, 23, 24]. It was shown 20, 21] that a significant improvement of the photoassociation rate compared to cw excitation can be obtained, since during the pulse the resonance condition is fulfilled for several vibrational levels $v^{\prime}$. However, the quantity which must be improved is 
the formation rate of ground state molecules rather than the photoassociation rate. One advantage of excitation with chirped pulses is that the chirp parameter can be chosen to modify the shape of the vibrational wave packet formed in the potential $V_{\text {exc }}$. This may improve the efficiency of the stabilization step, and thus the formation rate of ground state molecules.

The aim of the present work is therefore to suggest a two-color experiment using short laser pulses and to investigate how efficiently a second pulse can transfer the population to the bound vibrational levels of the ground state. Starting from the vibrational wave packet formed in the excited state by photoassociation, we introduce a second pulse which is delayed in time from the first pulse. The time delay is chosen such that the wave packet has reached the vicinity of the inner turning point, where a vertical radiative transition to the ground state is expected to be efficient. The parameters of the second pulse, as well as the time delay between the two pulses are varied to improve the efficiency of the transfer, with two different goals: to either maximize the total population transfer, or to selectively populate one vibrational level. The paper is organized as follows: In Sec. [II we recall the main results of previous work on photoassociation with chirped laser pulses. The two-color scheme is introduced in Sec. III] and the best choice for the dump pulse discussed by considering the Franck-Condon overlap between the vibrational wave packet in the excited state and wave functions of the bound levels in the ground state. The results for maximum population transfer are presented in Sec. [V] while Sec. $\nabla$ reports on the selective population of a single level. Finally Sec. VI concludes.

\section{CHIRPED PULSE PHOTOASSOCIATION IN $\mathrm{CS}_{2}$ AND CREATION OF A FOCUSSED VIBRATIONAL WAVE PACKET}

We briefly summarize the main results of Refs. 20, 21. for the photoassociation of cold cesium atoms by chirped laser pulses. The main difference to $\mathrm{cw}$ excitation lies in two facts: Several levels can be populated in the photoassociation step, and the vibrational wave packet can be shaped in order to allow for spatial "focussing", as described below. We start from a transform-limited pulse with Gaussian envelope, hereafter referred to as pump pulse, with maximum at $t=t_{P}$, and with full width at half maximum of the temporal intensity profile (FWHMI) $\tau_{L}=15 \mathrm{ps.} \mathrm{Chirping} \mathrm{the} \mathrm{pulse} \mathrm{stretches} \mathrm{the}$ FWHMI to $\tau_{C}=34.8 \mathrm{ps}$ and decreases the maximum intensity $I_{L} \rightarrow I_{L} \frac{\tau_{L}}{\tau_{C}}\left(I_{L}=120 \mathrm{~kW} \mathrm{~cm}{ }^{-2}\right)$, conserving the energy carried by the field. Moreover, $98 \%$ of this energy is carried during the time window $\left[t_{P}-\tau_{C}, t_{P}+\tau_{C}\right]$. We consider the reaction

$$
\begin{aligned}
& 2 \mathrm{Cs}(6 s, F=4)+\hbar\left[\omega_{L}+\chi_{P}\left(t-t_{P}\right)\right] \rightarrow \\
& \mathrm{Cs}_{2}\left(0_{g}^{-}\left(6 s^{2} S_{1 / 2}+6{ }^{2}{ }^{2} P_{3 / 2} ; v^{\prime}, J^{\prime}=0\right)\right)
\end{aligned}
$$

where the carrier frequency $\omega_{L}$ is chosen red-detuned relative to the $\mathrm{D}_{2}$ atomic line, at resonance with the $v^{\prime}=v_{L}^{\prime}=98$ level in the external well of $V_{\text {exc }}$ which is bound by $2.65 \mathrm{~cm}^{-1}$. $\chi_{P}$ is the linear chirp rate in time domain, chosen negative and equal to $-0.025 \mathrm{~cm}^{-1} \mathrm{ps}^{-1}$. The parameters of the pump pulse, labelled $\mathcal{P}_{-}$are recalled in Table I We will also consider other possibilities for the pump pulse, with positive or zero chirp, or with a smaller detuning: such pulses are respectively labelled $\mathcal{P}_{+}, \mathcal{P}_{0}$ and $\mathcal{P}_{-}^{122}$. The latter pulse has a central frequency resonant with the excited level $v^{\prime}=122$. The parameters for $\mathcal{P}_{-}^{122}$ have been chosen to ensure the focussing condition without populating continuum levels in the photoassociation step. Note that in order to compare to earlier papers, we have also considered another the temporal width,

$$
\begin{array}{r}
\tau^{P}=\sqrt{2} \tau_{L}, \\
\tau_{C}^{P}=\sqrt{2} \tau_{C}, \\
f_{P}=\tau_{C} / \tau_{L}=\tau_{C}^{P} / \tau^{P},
\end{array}
$$

where $\tau^{P}$ is now defined as the full width at half maximum of the electric field profile (FWHME) of the transform-limited pulse, while in Refs. 20, 21 $\tau_{L}$ was defined with respect to intensity (FWHMI). $f_{P}$ is the stretching factor due to chirping. During the time window, the central frequency is swept from $\omega_{L}-\chi \tau_{C}$ to $\omega_{L}+\chi \tau_{C}$ such that the resonance condition is satisfied for 15 vibrational levels around $v_{L}^{\prime}=98$ in the energy range $2 \hbar|\chi| \tau_{C}=2 \times 0.87 \mathrm{~cm}^{-1}$. Depending upon the sign of the chirp, the frequency is increasing $\left(\chi_{P}>0\right)$ or decreasing $\left(\chi_{P}<0\right)$ with time.

The dynamics of the photoassociation process was studied by numerical solution of the time-dependent Schrödinger equation describing the motion of wave packets in the ground state potential $a^{3} \Sigma_{u}^{+}(6 s+6 s)\left(V_{\text {ground }}\right)$ and in the $0_{g}^{-}\left(6 s^{2} S_{1 / 2}+6 p^{2} P_{3 / 2}\right)$ excited potential $\left(V_{\text {exc }}\right)$ coupled by the electromagnetic field. The initial state is represented by a stationary continuum wave function describing the relative motion ( $s$-wave) of two colliding $\mathrm{Cs}(6 \mathrm{~s}, \mathrm{~F}=4)$ atoms, with an initial kinetic energy of $54 \mu \mathrm{K}$. The wave packet in the excited potential $V_{\text {exc }}$ is analyzed by projection onto the vibrational wave functions of the $v^{\prime}$ levels in the external well. The main results of the calculations are:

- During the pulse, many vibrational levels are populated. However, after the pulse, the population remains only in the 15 vibrational levels resonantly excited around $v_{L}^{\prime}=98$. This defines a photoassociation window in the energy domain, for the levels located between $v_{1}^{\prime}=106$ and $v_{2}^{\prime}=92$, bound by $1.74 \mathrm{~cm}^{-1}$ and $3.57 \mathrm{~cm}^{-1}$, respectively.

- In the impulsive approximation [25], assuming that the relative motion of the two atoms is frozen during the pulse, it is possible to analyze the numerical results by a two-state model at each internuclear distance $R$. While the frequency of the laser 


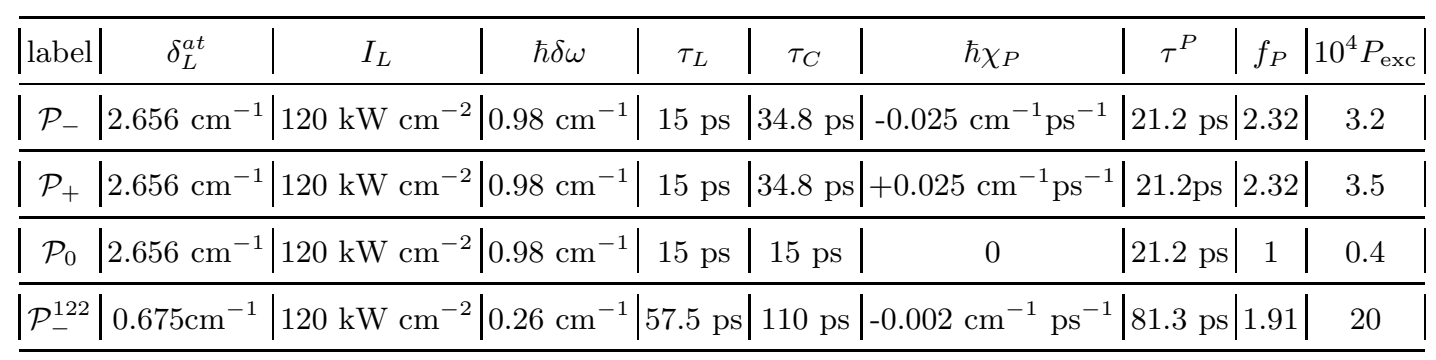

TABLE I: Parameters for the main pump pulse $\mathcal{P}_{-}$considered in Refs. [20, 21]: detuning $\delta_{L}^{a t}$, maximum intensity $I_{L}$, energy range associated to the spectral width $\hbar \delta \omega$, temporal widths (FWHMI) $\tau_{L}$ and $\tau_{C}$, linear chirp parameter $\chi_{P}$; new parameters used in the present work are the temporal width (FWHME) before chirping $\tau^{P}$, and stretching factor $f_{P}$ (see Eq. (2) in text). Also indicated are other pump pulses with different chirp or different detuning. In the last column, we indicate the population transferred to the excited state by the photoassociation pulse.

is swept from $\omega_{L}-\chi_{P} \tau_{C}$ to $\omega_{L}+\chi_{P} \tau_{C}$, the crossing point $R_{c}(t)$ of the two field-dressed potential curves is moving in the $\left[R_{\min }, R_{\max }\right]$ range. Assuming that the resonance condition corresponds to a vertical transition at $R=R_{c}(t)$, this defines a photoassociation window in the $R$ domain: in the example chosen, $R_{\min }=85$ and $R_{\max }=107 \mathrm{a}_{0}$. Within this range of distances, all the population of the initial continuum level can be adiabatically transferred to the excited state.

- In the excited state, the levels $v^{\prime}$ which are populated have a classical outer turning point $R_{\text {out }}^{v^{\prime}}$ in the $\left[R_{\min }, R_{\max }\right]$ range, and the two-state model predicts that the population transfer occurs around the time $t_{v^{\prime}}$ when $R_{\mathrm{out}}^{v^{\prime}}=R_{c}\left(t_{v^{\prime}}\right)$. For a negative chirp, the position of the crossing point varies from $R_{\max }$ to $R_{\min }$, so that the upper levels are populated first (see Fig. 1). For the pump pulse considered, the level $v_{1}^{\prime}=106$ is populated at the beginning of the time window $\left(t_{1} \sim\left(t_{P}-\tau_{C}\right)\right.$, the level $v_{2}^{\prime}=92$ is populated at the end $\left(t_{2} \sim t_{P}+\tau_{C}\right)$, i.e. 70 ps later. It is the opposite for a positive chirp, choosing the $\mathcal{P}_{+}$pulse for photoassociation.

- The total probability of population transfer to the excited state is reported in the last column of Table [1 The absolute value is discussed in Refs. 20, 21], the important result for the present paper being that the population obtained with $\mathcal{P}_{0}$ is increased by a factor 8 when a chirped pulse $\mathcal{P}_{-}$ is considered, and by a factor of 6 when the pulse $\mathcal{P}_{-}^{122}$, with a smaller detuning, is used.

- After the end of the pulse, the vibrational wave packet $\Psi_{\text {exc }}(R, t)$ in the excited state is moving from the outer classical turning point toward shorter distances (see Fig 1). We analyze it through partial wave packets, with main components corresponding to the various levels $v^{\prime}$. Each one is reaching the inner turning point at a time which

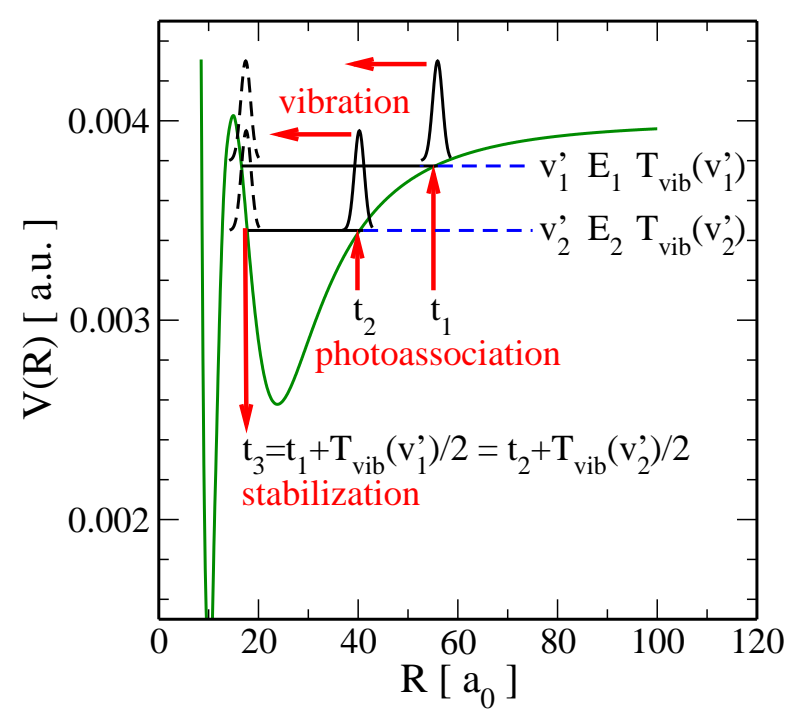

FIG. 1: (Color online) Photoassociation with a chirped pulse: For negative chirp, the level $v_{1}^{\prime}$ is populated at the beginning $\left(t_{1}\right)$, while $v_{2}^{\prime}$ is populated at the end $\left(t_{2}\right)$ of the time window characterizing the photoassociation pulse. The magnitude of the chirp can be chosen such that, after half a vibration period $T_{v i b}$, the partial wave packets arrive at the potential barrier at the same time $t_{3}$. This time $\left(t_{3}\right)$ is optimal for radiative stabilization, with a second pulse, toward bound ground state levels.

depends upon the excitation time $t_{v^{\prime}}$ and the vibrational period $T_{v i b}\left(v^{\prime}\right)$. In the photoassociation window considered here, the vibrational periods are in the range $350-196 \mathrm{ps}$, yielding $\frac{1}{2} T_{\mathrm{vib}}\left(v_{1}^{\prime}\right)=175 \mathrm{ps}$ and $\frac{1}{2} T_{\mathrm{vib}}\left(v_{2}^{\prime}\right)=98 \mathrm{ps}$, respectively. For a negative chirp, we may choose the parameters of the pulse such as to create partial vibrational wave packets which reach the inner turning point approximately at the same time $t_{3} \approx t_{1}+1 / 2 T_{\text {vib }}\left(v_{1}^{\prime}\right) \approx$ $t_{2}+1 / 2 T_{\mathrm{vib}}\left(v_{2}^{\prime}\right)$. For long range molecules, the vibrational period is known to obey a scaling law [26], 
and it is easy to choose the chirp parameter such that all the partial wave packets in the intermediate levels $v_{2}^{\prime} \leq v^{\prime} \leq v_{1}^{\prime}$ satisfy 21 .

$$
t_{v^{\prime}}\left(\chi_{P}\right)+\frac{T_{\mathrm{vib}}\left(v^{\prime}\right)}{2} \approx t_{P}+\frac{T_{\mathrm{vib}}\left(v_{L}^{\prime}\right)}{2}=t_{3} .
$$

Focussing of the wave packet is then obtained at time $t_{3}$, after half a vibrational period. In contrast, for a positive chirp the lower levels are populated first, $t_{2}<t_{1}$, and a spreading of the vibrational wave packet is expected.

- Several authors in the past have suggested ways of designing laser fields for the generation of spatially squeezed molecular wave packets [27, 28]. Since they considered low vibrational levels, a numerical optimization procedure appeared to be necessary. Here, we are dealing with long range molecules, where the motion is controlled by the asymptotic potential, and the availability of scaling laws makes the optimization easier, as for atomic Rydberg wave packets 29].

- We should note that in the chosen example, the vibrational progression and the scaling law of the vibrational period are slightly perturbed due to a tunneling effect for the levels in the vicinity of $v^{\prime}=96[20,30]$. This leads to a focussing time $t_{3}=t_{P}+135 \mathrm{ps}$, slightly different from $\frac{1}{2} T_{\mathrm{vib}}\left(v^{\prime}=\right.$ $98)=125$ ps. Moreover, the early population of levels which are perturbed by tunneling leads to population transfer to the inner well of the $0_{g}^{-}\left(6 s+6 p_{3 / 2}\right)$ excited potential. This tunneling effect is not a general phenomenon, but very specific to the cesium $0_{g}^{-}$molecular curve, and to the value chosen for the detuning. A detailed investigation would require consideration of two coupled channels in the region of the inner potential [30], beyond the scope of this work.

In the present calculations, we start from the wave packet in the excited state and investigate how the population can efficiently be transferred to vibrational levels of the ground state by the second pulse. As in our previous work, this is achieved by studying the wave packet dynamics by numerical solution of the time-dependent Schrödinger equation (cf. Ref. 20, 21] for an overview over numerical methods and Appendix A for a discussion of the representation of continuum states).

\section{THE TWO-COLOR SCHEME}

The scheme we suggest is illustrated in Fig. 2 (left), where we have represented, at different times, the wave packet created by the negatively chirped picosecond pulse $\mathcal{P}_{-}$. We consider a vibrational wave packet $\Psi_{\text {exc }}(R, t=$ $\left.t_{P}+t_{\mathrm{dyn}}\right)$, which has evolved on the excited state potential for time $t_{\mathrm{dyn}}$ after the maximum of the pump pulse
$t_{P}$. In order to discuss directly transfer probabilities, we will renormalize the wave packet before applying the dump pulse, such that at any time $<\Psi_{\text {exc }} \mid \Psi_{\text {exc }}>=1$. The variation of the photoassociation probability as reported in the last column of Table @ will be reconsidered in the conclusion.

\section{A. The Franck-Condon overlap and its time-dependence: choice of the target vibrational level in the stabilization step}

We first compute the Franck-Condon overlap matrix elements $\left|\left\langle\Psi_{\text {exc }}\left(t_{\mathrm{dyn}}\right) \mid \varphi_{v^{\prime \prime}}^{g}\right\rangle\right|^{2}$ of the excited state wave packet with the bound ground state eigenfunctions (cf. Fig. 2 right). The results markedly depend upon $t_{\mathrm{dyn}}$, and so does the probability to form stable molecules. For instance, just after the photoassociation pulse, at time $t_{P}+40 \mathrm{ps} \sim t_{P}+\tau_{C}$ (circles in Fig. 2 right), the FranckCondon overlap factors are negligible for most bound levels of the ground state, except for the last level $v^{\prime \prime}=53$ which is very loosely bound $\left(5 \times 10^{-6} \mathrm{~cm}^{-1}[20]\right)$. In contrast, half a vibrational period later, at time $t_{P}+135 \mathrm{ps}$ when the wave packet has moved to the inner turning point (diamonds in Fig. 2 right), a significant overlap is obtained with various deeply bound levels of the ground state, in particular with $v^{\prime \prime}=6,14,20$. The large overlap with $\varphi_{v^{\prime \prime}=14}^{g}(R)$ can be explained by the coincidence of the inner classical turning point of the level $v^{\prime}=92$ and the outer turning point of $v^{\prime \prime}=14$, such that $v^{\prime}=92 \rightarrow$ $v^{\prime \prime}=14$ is a Franck-Condon transition. This large value is restricted to a short $(\sim \pm 2 \mathrm{ps})$ time window around $t_{P}+135 \mathrm{ps}$ as illustrated in Fig. 3. where the timedependence of the Franck-Condon overlap is shown. Note that the overlap with $v^{\prime \prime}=6$ is due to population in the inner well of $V_{\text {exc }}$ due to tunneling [30]. From Fig. 3] we can indeed see that this population remains trapped at the considered timescale.

The above mentioned large overlap with the last vibrational level of the ground state just after the photoassociation pulse $\left(t-t_{P} \leq \tau_{C}\right)$ is seen to reappear after one vibrational period, when the wave packet reaches again the outer turning point. However, we will not choose one of the last bound levels of the ground state as our target vibrational level $v^{\prime \prime}$, since a significant population is already achieved in a one-color experiment [20, 21], making the implementation of a two-color scheme superfluous. In fact, the specific advantage of a pump-dump experiment is the possibility of stabilization into deeply bound levels in the ground state. We will therefore focus on populating the level $v^{\prime \prime}=14$ with binding energy $E_{\mathrm{b}}=113 \mathrm{~cm}^{-1}$, and we will consider a second (or dump) pulse $\mathcal{D}$ with parameters adapted to efficient population transfer toward $v^{\prime \prime}=14$, in particular with a time delay close to 135 ps. 

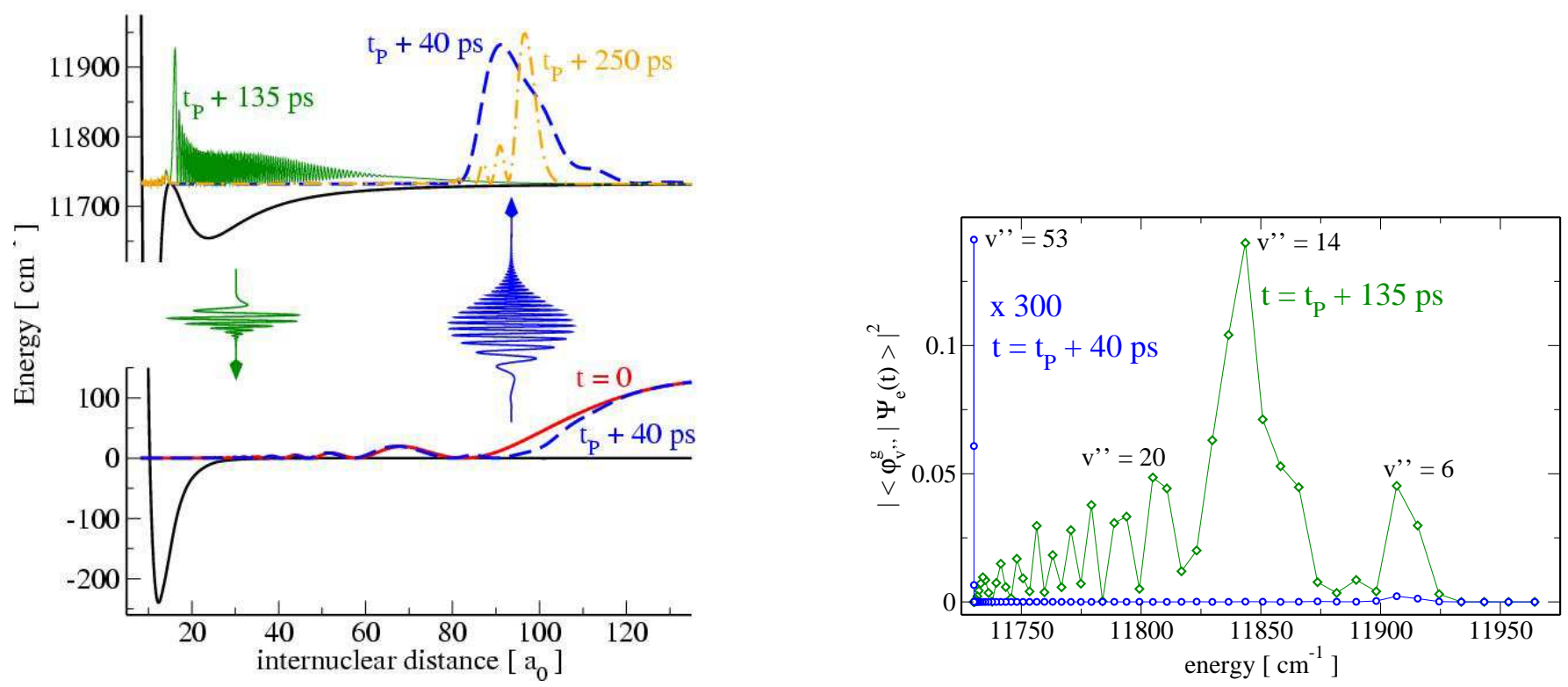

FIG. 2: (Color online) left: The two-color scheme: A chirped picosecond pulse $\mathcal{P}_{-}$(see Table ) excites a wave packet in the electronically excited state. A second, shorter pulse $\mathcal{D}$ (see Tables II IV) dumps this wave packet to the electronic ground state. right: Franck-Condon overlap between the time-dependent wave packet (population normalized to one, see text) in the excited state, and ground state vibrational levels at times $t=t_{P}+40$ ps, just after the photoassociation pulse (blue circles) and at $t=t_{P}+135 \mathrm{ps} \approx t_{\text {in }}$ (green diamonds), when the wave packet is focussed at the inner barrier; $t_{P}$ is the time when the photoassociation pulse is maximum. When the wave packet is focussed at the inner turning point, there is a maximum overlap with the level $v^{\prime \prime}=14$ of the ground state.

\section{B. Choice of parameters for the second pulse}

For the dump pulse $\mathcal{D}$, we define a central frequency $\omega_{L}^{D}$, a maximum intensity $I_{L}$, a duration (FWHME) of the transform-limited pulse $\tau_{D}$, the time of maximum amplitude $t_{D}$, and a stretching factor $f_{D}$ characterizing the strength of the chirp. The choice of the second color is dictated by the choice of the target levels. For $v^{\prime \prime}=14$ (cf. Fig. 2 right), we have chosen a central frequency $\omega_{L}^{D}=11843.5 \mathrm{~cm}^{-1}$, which is resonant with $v^{\prime \prime}=14$ for a binding energy of the excited state wave packet equal to $2.1 \mathrm{~cm}^{-1}$. This binding energy, computed as $\left\langle\Psi_{\text {exc }}(R, t)\left|\hat{T}+V_{\text {exc }}\right| \Psi_{\text {exc }}(R, t)\right\rangle$, where $\hat{T}$ is the kinetic energy operator, stays time-independent once the photoassociation pulse is over. It is found $0.5 \mathrm{~cm}^{-1}$ smaller than the binding energy of the level $v^{\prime}=v_{L}^{\prime}=98$ which is resonant with the central frequency of the pump pulse. This difference reflects the non-symmetric population of the $v^{\prime}$ levels relative to $v_{L}^{\prime}$, the photoassociation process being much more efficient for the upper levels.

The spectral width of the second pulse $\mathcal{D}$ is limited by the requirement that mostly bound levels should be populated in the ground state. Since $v^{\prime \prime}=14$ is bound by 113 $\mathrm{cm}^{-1}$, the energy spread of the vibrational wave packet in the excited state can be neglected and the maximum spectral width is estimated as $2 \times 113 \mathrm{~cm}^{-1}$. Therefore, for short pulses $\mathcal{D}$ (FWHME $\tau^{D} \leq 93 \mathrm{fs}$ ), population transfer to the continuum is to be expected leading to the formation of pairs of "hot" atoms. This conclusion relies on overlap integrals and needs to be corrected by the possible energy dependence of the transition dipole moment $\mu(R)$. Finally, if we wish to selectively excite the level $v^{\prime \prime}=14$, the spectral width is limited by the vibrational energy splitting around $v^{\prime \prime}=14$ which is about $7 \mathrm{~cm}^{-1}$. The typical pulse temporal width $\tau^{D}$ of the dump pulse should therefore be larger than 2 ps. In the following, we will discuss two schemes to form ground state molecules:

1. For short pulses in the 10-300 fs range, important population transfer to several bound levels of the ground electronic state can be obtained (cf. SecIV]. The goal is then to maximize the total population transfer. The intensity, duration and possibly stretching factor, i.e. chirp, of the $\mathcal{D}$ pulses will be systematically varied.

2. For longer pulses in the picosecond domain, transfer of population to a single vibrational level, namely $v^{\prime \prime}=14$, is possible (see Sec V). The goal is then to optimize selective population transfer to a chosen level.

The third parameter is the time-delay between the two pulses, defined as $t_{D}-t_{P}$. The discussion can be guided by the following arguments, illustrated in Fig. 4]

- First, we can use a simple model for the choice of the time delay $t_{D}-t_{P}$. Considering the wave packet $\Psi_{\text {exc }}\left(R, t_{D}\right)$, it is possible to estimate an 


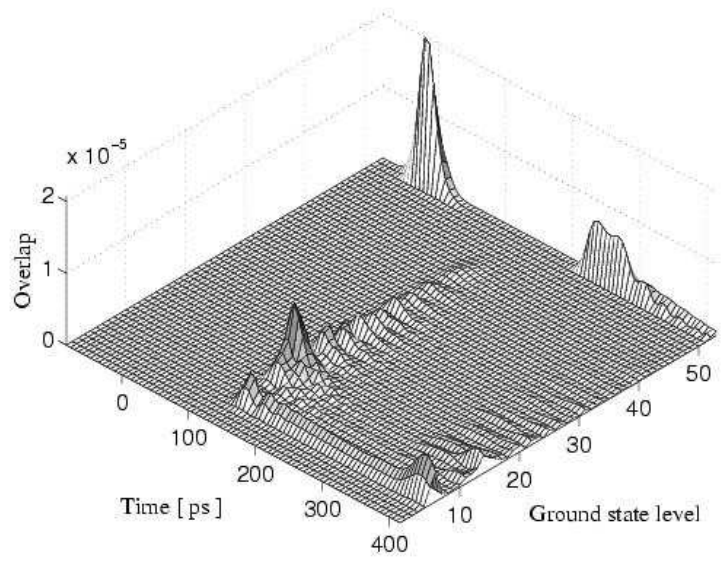

FIG. 3: A two-dimensional plot of the Franck-Condon overlap of Fig. 2(b) between the time-dependent wave packet in the excited state and all bound vibrational levels of the ground state. $t_{P}$ corresponds to $t=0$. It is clear that the overlap with deeply bound ground state levels (around $v^{\prime \prime}=14$ ) is maximum when focussing of the excited state wave packet occurs, $135 \mathrm{ps}$ or half a vibrational period after $t_{P}$. In contrast, when the wave packet is at its outer turning point just after the PA pulse and again at about $t_{P}+250 \mathrm{ps}$ (one vibrational period $T_{v i b}$ ), the overlap with only the last bound levels is significant. Moreover, tunneling to and trapping of population in the inner potential well lead to overlap with ground state levels around $v^{\prime \prime}=6$ : this is specific to the cesium $0_{g}^{-}\left(P_{3 / 2}\right)$ state and to the value chosen for the detuning. Note the stability of this population during half a vibrational period.

upper limit to the probability of forming molecules from the sum over the Franck-Condon overlap matrix elements $\left|\left\langle\Psi_{\text {exc }}\left(R, t_{D}\right) \mid \varphi_{v^{\prime \prime}}^{g}\right\rangle\right|^{2}$. After a short and weak dump pulse at time $t_{D}$, the wave packet created in the ground state is estimated by

$$
\left|\psi_{g}(t)\right\rangle=\varlimsup_{E} \mu \varepsilon(E)\left\langle\varphi_{E}^{g} \mid \psi_{e}\left(t_{D}\right)\right\rangle \mathrm{e}^{-\frac{i}{\hbar} E\left(t-t_{D}\right)}\left|\varphi_{E}^{g}\right\rangle,
$$

where $£_{E}$ denotes jointly the sum over bound levels with eigenenergies $E=E_{i}$ and the integral over continuum states with energy $E$, while $\varepsilon(E=\hbar \omega)$ is the Fourier transform of the field $\varepsilon(t)$ and $\mu$ the dipole moment, assumed to be $R$-independent. Eq. (4) implies that vibrational levels and continuum states which are resonant within the bandwidth $\varepsilon(\omega)$ of the pulse are excited. The population transfer is proportional to the time-dependent Franck-Condon factors discussed above. Assuming a $\pi$-pulse (i.e. a pulse which leads to population inversion) with bandwidth covering all ground state vibrational levels, the maximum probability is given by $\left|\left\langle\psi_{g}\left(t_{D}\right) \mid \psi_{g}\left(t_{D}\right)\right\rangle\right|^{2}$, restricting the sum in Eq. (4) to the bound levels. The highest probability, 0.2 , is expected for a delay of $135 \mathrm{ps}$ (cf. Fig. (4), when the wave packet $\Psi_{\text {exc }}\left(R, t_{P}+135\right)$ is

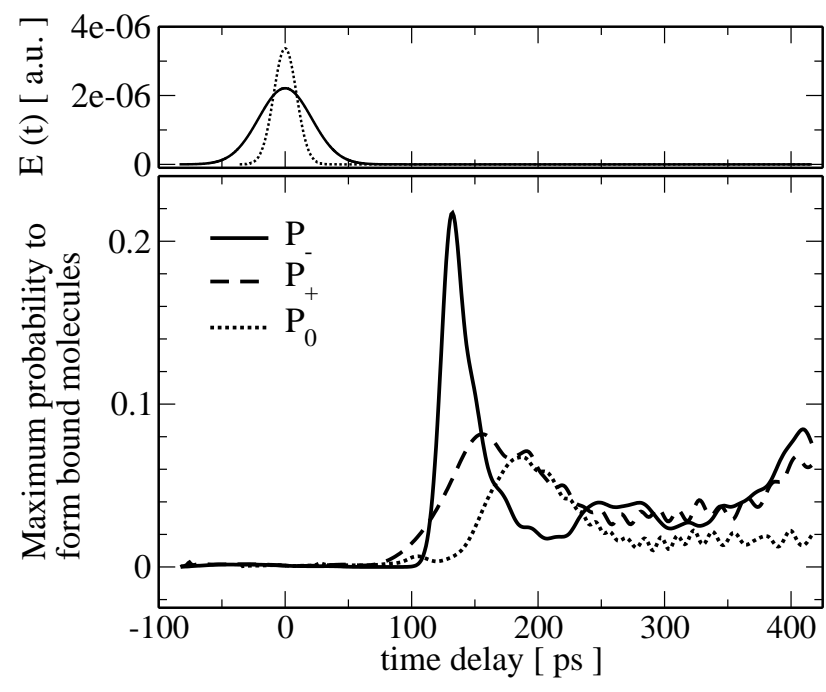

FIG. 4: (top) The photoassociation pulses $\mathcal{P}_{ \pm}, \mathcal{P}_{0}$. (bottom) The upper limit to the probability of forming bound molecules is given by the sum over Franck-Condon overlap matrix elements between the time-dependent excited state wave packet (assumed normalized to one) and all the bound levels of the ground state. The highest probability of about $20 \%$ is expected for the focussed wave packet (at time $t=t_{P}+135 \mathrm{ps}$ ).

focussed after half a vibrational period. Note the relatively short time window in which the probability is maximum. A secondary maximum is visible after one vibrational period ( 250 to $300 \mathrm{ps)}$ ) when the last levels are populated: much less population can then be transferred. Note also the revival at $t_{D} \sim 400 \mathrm{ps}$, after one and a half vibrational period, with a larger width. The advantage of focussing is further manifested by comparing to the Franck-Condon overlaps of wave packets created by the pump pulses $\mathcal{P}_{+}$and $\mathcal{P}_{0}$. The maximum probability is now less than $10 \%$, instead of $20 \%$ for the focussed wave packet, and it is spread during a larger time window.

- Next, we report time-dependent calculations for various dump pulses where the field is treated nonperturbatively and we start from a focussed wave packet. Intensity and spectral width are varied (see below Sec. IVB in order to check the validity limits of the above mentioned simple model which relies on the impulsive approximation and weak fields. Then a chirp of the dump pulse is introduced to obtain a robust scheme for population inversion (cf. Sec. IVC). The dependence on the time delay is studied in Sec.IVD We furthermore present calculations starting from vibrational wave packets created with other photoassociation pump pulses such as $\mathcal{P}_{+}, \mathcal{P}_{0}$ (Sec. IVE) and considering a smaller detuning (Sec. IVF). 


\section{STABILIZATION WITH A BROADBAND PULSE POPULATING MANY VIBRATIONAL LEVELS OF THE GROUND STATE}

\section{A. Concept of a molecular $\pi$ pulse}

When the aim is to transfer as much population to the ground state as possible, the discussion of the pulse parameters is inspired by the arguments on population inversion in molecular electronic states developed in Refs. 22, 31]. The basic idea is that for pulses which are short on the timescale of the vibrational dynamics, the wave packet does not move during the pulse (impulsive approximation). The molecular system can then be reduced to an effective two-level system, and it is possible to find a $\pi$-pulse which leads to total population transfer. A $\pi$ pulse is defined as a pulse (assumed here to be transformlimited and with Gaussian envelope) whose Rabi angle,

$$
\begin{aligned}
\vartheta & =\int_{-\infty}^{+\infty} \mu \epsilon(t) d t=\int_{-\infty}^{+\infty} \mu \epsilon_{0} \exp \frac{-\sqrt{2 \ln 2} t^{2}}{\tau^{D}} d t \\
& =\mu \sqrt{\frac{\pi}{\ln 2}} \tau^{D} \epsilon_{0}
\end{aligned}
$$

is equal to an odd multiple of $\pi$. A pulse with Rabi angle equal to an even multiple of $\pi$ induces zero population transfer. Introducing a chirp enforces adiabatic following conditions for all Rabi angles $>\pi$ and therefore suppresses the oscillations in population transfer as a function of the Rabi angle [22, 24, 31].

\section{B. Inducing maximum population inversion}

The time-dependent Schrödinger equation describing the evolution of the wave packets $\Psi_{\text {exc }}(R, t)$ and $\Psi_{\mathrm{g}}(R, t)$ in the excited and in the ground state, coupled by the radiative field of the dump pulse is solved numerically. Our treatment of the field is non-perturbative (cf. Refs. [20, 21]) and for intensities corresponding to Rabi angles $\vartheta>\pi$ deviations from the estimate of Eq. (4) are expected. Deviations will also occur if the impulsive approximation ceases to be valid, i.e. for longer pulses. In this case, nuclear motion during the pulse cannot be neglected and the wave packet is not simply projected to the electronic ground state.

For all calculations presented in this section, the parameters of the short dump pulses are recalled in Table The initial condition is given by the focussed excited state wave packet at $t=t_{P}+135 \mathrm{ps}$, normalized to one such that population corresponds directly to probability. The propagation is carried out in the time interval $\left[t_{0}=t_{D}-4 \sigma, t_{\text {final }}=t_{D}+4 \sigma\right]$ with $\sigma=f_{D} \tau^{D} / 2 \sqrt{2 \ln 2}$. The final population in the ground state $P_{g}\left(t_{\text {final }}\right)$ is given by $P_{g}\left(t_{\text {final }}\right)=\left|\left\langle\Psi_{\mathrm{g}}\left(t_{\text {final }}\right) \mid \Psi_{\mathrm{g}}\left(t_{\text {final }}\right)\right\rangle\right|^{2}$ while the probability to form bound molecules corresponds to $P_{g}^{\text {bound }}=$ $\sum_{i}\left|\left\langle\varphi_{i}^{g} \mid \Psi_{\mathrm{g}}\left(t_{\text {final }}\right)\right\rangle\right|^{2}$.

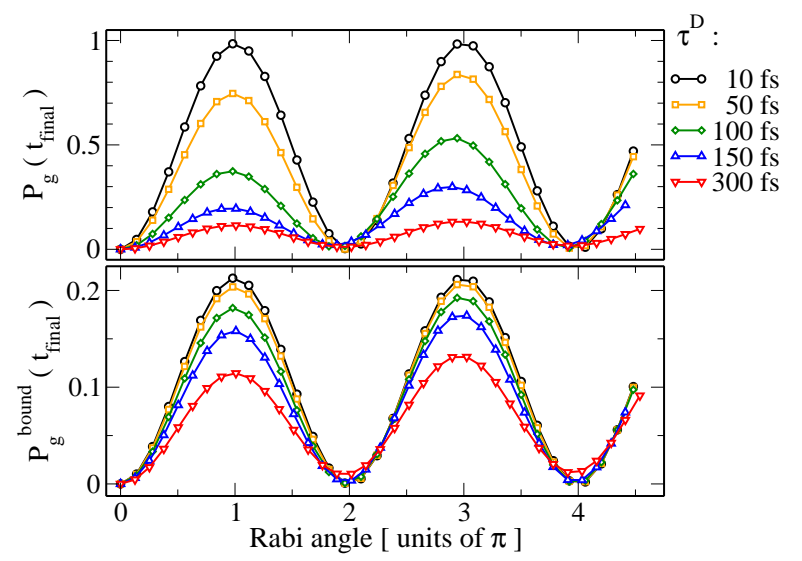

FIG. 5: (Color online) The final ground state population (top) and the final population of bound levels in the ground state (bottom) after a transform-limited dump pulse (see Table II) as a function of the Rabi angle, i.e. intensity. For all pulse durations shown, we find pronounced Rabi oscillations, i.e. the approximation of an effective two-level systems holds. Very short pulses (10 fs) are able to induce complete population inversion, but the population which ends up in bound levels is limited to about $20 \%$; the dump pulse is then dissociating $80 \%$ of the photoassociated molecules. Longer pulses (300 fs) populate only bound levels and no continuum states, since, within their bandwidth, only bound levels are on resonance.

In Fig. [ w we analyze the efficiency of the population transfer by varying the intensity of a transform-limited dump pulse for several durations $\tau^{D}$, in the range of 10$300 \mathrm{fs}$. The approximation of an effective two-level system is shown to hold for all pulse durations, since oscillations as a function of the Rabi angle are clearly manifested in the final state population $P_{g}\left(t_{\text {final }}\right)$ and in the population of bound levels $P_{g}^{\text {bound }}\left(t_{\text {final }}\right)$. The contrast of these oscillations is maximum for $\tau^{D}=10 \mathrm{fs}$, where total population inversion is obtained with $P_{g}\left(t_{\text {final }}\right)$ oscillating between 0 and 1 . For longer pulses $\left(\tau^{D}=300 \mathrm{fs}\right)$, only $10 \%$ of the population is transferred to the ground state. However, an analysis of the wave packet in the ground state shows that for the shorter pulses, the population is spread between continuum and bound levels. The probability to form bound molecules is at most $20 \%$ $\left(\tau^{D}=10 \mathrm{fs}\right)$, so that $80 \%$ of the photoassociated molecules are dissociated by the dump pulse, giving pairs of hot atoms which usually leave the trap. With longer pulses, due to their narrower frequency bandwith, the transfer to continuum levels is no longer possible: for $\tau^{D}=300$ fs the $11 \%$ population transferred to the ground state is totally stabilized into bound levels. The maximum population transfer and its efficiency are reported in Table III demonstrating that in the intermediate range of pulse widths, up to $100 \mathrm{fs}$, a stabilization of $\sim 20 \%$ of the photoassociated molecules is to be expected.

The possibility of stabilizing $20 \%$ of the photoassociated molecules is very appealing. However, the vibrational distribution of the population transferred to 


\begin{tabular}{|c|c|c|c|c|c|c|}
\hline$\omega_{L}^{D}$ & $\hbar \delta \omega_{D}$ & $\tau^{D}$ & $f_{D}$ & $\chi_{D}$ & delay $t_{D}-t_{P} \mid$ Figs. \\
\hline $11843.5 \mathrm{~cm}^{-1}\left|2000-70 \mathrm{~cm}^{-1}\right| 10-300 \mathrm{fs}$ & 1 & 0 & $135 \mathrm{ps}$ & 56 \\
\hline $11843.5 \mathrm{~cm}^{-1}\left|200,70 \mathrm{~cm}^{-1}\right| 100,300 \mathrm{fs}$ & $2,5,10$ & $120,21.7,5.52 \mathrm{ps}^{-2}$ & $135 \mathrm{ps}$ & |7to 110 \\
\hline $11845.0 \mathrm{~cm}^{-1}\left|200,70 \mathrm{~cm}^{-1}\right| 100,300 \mathrm{fs}$ & 1 & 0 & $135 \mathrm{ps}$ & 12 \\
\hline
\end{tabular}

TABLE II: Parameters for the short dump pulses considered in Sec. IV central frequency $\omega_{L}^{a t}$, temporal width $\tau^{D}$ (FWHME), stretching factor $f_{D}$, linear chirp parameter $\chi_{D}$, delay between the maxima of dump and pump pulse $t_{D}-t_{P}$. The corresponding figures are reported in the last column.

\begin{tabular}{|l|c|c|c|c|c|}
\hline$\tau^{D}[\mathrm{fs}]$ & 10 & 50 & 100 & 150 & 300 \\
\hline$P_{\mathrm{g}}$ & 0.984 & 0.746 & 0.374 & 0.195 & 0.114 \\
\hline$P_{\mathrm{g}}^{\text {bound }}$ & 0.212 & 0.208 & 0.180 & 0.158 & 0.114 \\
\hline$P_{\mathrm{g}}^{\text {bound }} / P_{\mathrm{g}}$ & 0.216 & 0.273 & 0.487 & 0.811 & 0.999 \\
\hline
\end{tabular}

TABLE III: Ground state populations after a dump $\pi$-pulse starting from a unity normalized wave packet in the excited state; ratio of population in bound levels to overall ground state population after $\pi$-pulse (cf. Fig. 5)

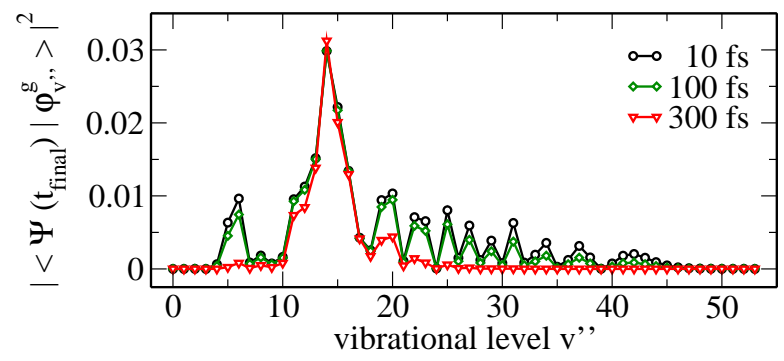

FIG. 6: (Color online) The vibrational distribution of ground state wave packets after $\pi$-pulses (cf. Fig. 5): The distributions after a $10 \mathrm{fs}$ and $100 \mathrm{fs}$ pulse are almost identical to the Franck-Condon overlap of the focussed excited state wave packet with ground state bound levels. The narrower bandwidth of a 300 fs pulse allows for less levels to be populated (cut-off around $v^{\prime \prime}=10$ and $v^{\prime \prime}=25$ ); furthermore, no continuum states are excited (cf. Table III).

the ground state should also be discussed, since stable molecules should be formed in low vibrational levels. The wave packet created in the ground state after a $\pi$ pulse is analyzed through projection on the various $\varphi_{v^{\prime \prime}}^{g}(R)$ vibrational wave functions, cf. Fig. 6] For short enough pulses $\left(\tau^{D}=10 \mathrm{fs}, 100 \mathrm{fs}\right)$ the distribution follows the variation of the Franck-Condon overlap discussed above (cf. Fig. 2 right). The difference between the $10 \mathrm{fs}$ and $100 \mathrm{fs}$ pulses affects mostly the population of continuum states (not shown in the figure). Due to the narrower bandwith, the longer pulse with $\tau^{D}=300$ fs is only populating levels up to $v^{\prime \prime}=25$, and no continuum levels. In all cases the levels around $v^{\prime \prime}=14$ are preferentially populated. The efficiency of the stabilization process would of course be reduced by considering Rabi angles different from $\pi$. Therefore, for a given pulse duration, the scheme is very sensitive to the intensity of the dump pulse. This can be avoided by introducing chirped dump pulses 22, 24, 31].

\section{Chirping the dump pulse to suppress oscillations as a function of the Rabi angle}

In Fig. 7 we report numerical results obtained after chirping the dump pulse, starting from transform-limited pulses of $100 \mathrm{fs}$ and $300 \mathrm{fs}$, respectively. The streching factor $f_{D}$ is varied from 1 to 10 , and the linear chirp parameter $\chi_{D}$ is chosen to be negative. Note that the Rabi angles in Fig. 7 only characterize the pulse intensities, they are those of the corresponding unchirped pulses (the Rabi angle becomes complex for chirped pulses). Fig. 7 clearly shows that chirping suppresses the variation of the final population as a function of the Rabi angle. For $\tau^{D}=100$ fs and $f_{D} \geq 5$ complete population inversion is reached at high intensities due to non-perturbative effects such as power broadening (top left). However, as for the unchirped pulses, the population of bound levels of the ground state remains limited to about $20 \%$ (bottom left). The advantage of chirping consists in this value of $20 \%$ being obtained for a wide range of Rabi angles $(\pi$ to $4 \pi$ in our calculations). For $\tau^{D}=300 \mathrm{fs}$, nearly complete population inversion is achieved for stretching factors $f_{D}=2,5$, and the probability of populating bound levels becomes close to 20\%, as for the shorter pulses. The chirp parameter should, however, not be too large: for a strongly chirped pulse $\left(f_{D}=10\right)$ with corresponding transform-limited FWHM $\tau^{D}=300 \mathrm{fs}$, the population transfer decreases, and oscillations reappear. This is due to the breakdown of the effective two-level system approximation: With a pulse stretched to $3 \mathrm{ps}$, it is no longer justified to neglect the relative motion of the nuclei during the pulse.

In Fig. 8 we compare the final ground state population after chirped dump pulses with $\tau^{D}=300$ for positive and negative chirp. The stretching factor is again varied from 1 to 10 . For pulses with correspond- 


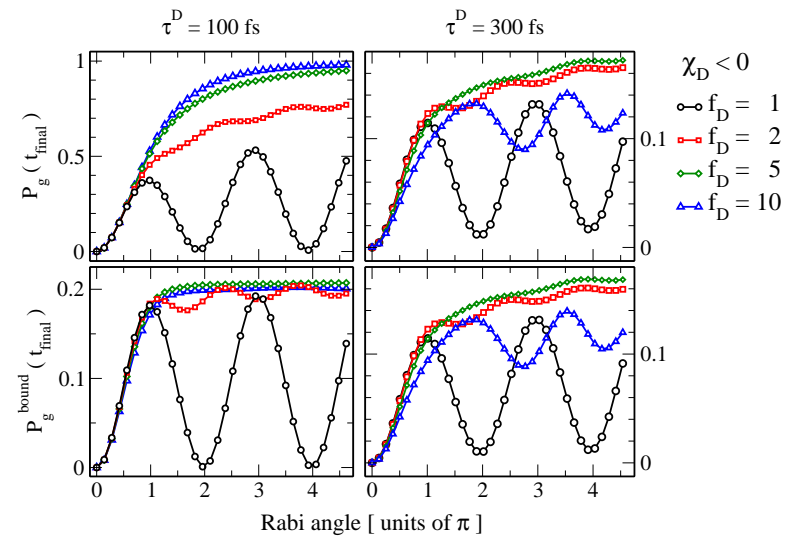

FIG. 7: (Color online) Overall ground state population (top) and population of bound ground state levels (bottom) after negatively chirped dump pulses as a function of the Rabi angle for different values of the stretch factor $f_{D}$. The duration of the transform-limited pulses is $\tau^{D}=100 \mathrm{fs}$ (left) and $\tau^{D}=$ $300 \mathrm{fs}$ (right). Results for an unchirped pulse are plotted for comparison (black circles).

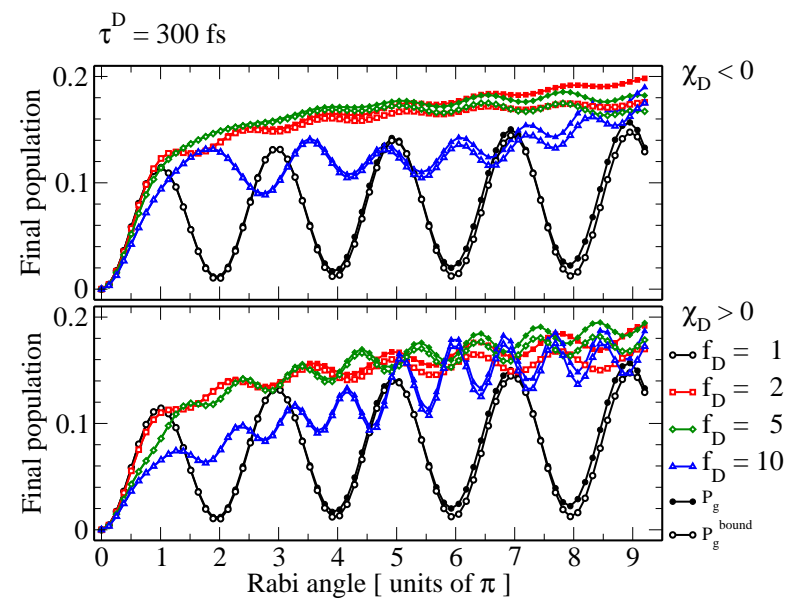

FIG. 8: (Color online) Overall ground state population (filled symbols) and population of bound ground state levels (open symbols) after negatively (top) and positively (bottom) chirped dump pulses with transform-limited FWHM of $300 \mathrm{fs}$ as a function of the Rabi angle for different values of the stretch factor $f_{D}$. The results for an unchirped pulse are plotted for comparison (black circles).

ing transform-limited FWHM $\tau^{D}=100$ fs, no difference between negative and positive chirp was observed (data not shown). For $\tau^{D}=300 \mathrm{fs}$, pulses with negative chirp perform slightly better than those with positive chirp. At high intensities $(\vartheta>4 \pi)$, differences between open and filled symbols are seen, i.e. even fairly narrow-bandwidth pulses start to excite continuum levels.

The effect of the chirp of the dump pulse on the final vibrational distribution in the ground state is analyzed in Fig. 9 A chirped pulse with intensity corresponding to $\vartheta=\pi$ (red squares in Fig. 9) leads to very a similar vibrational distribution as an unchirped $\pi$-pulse (black

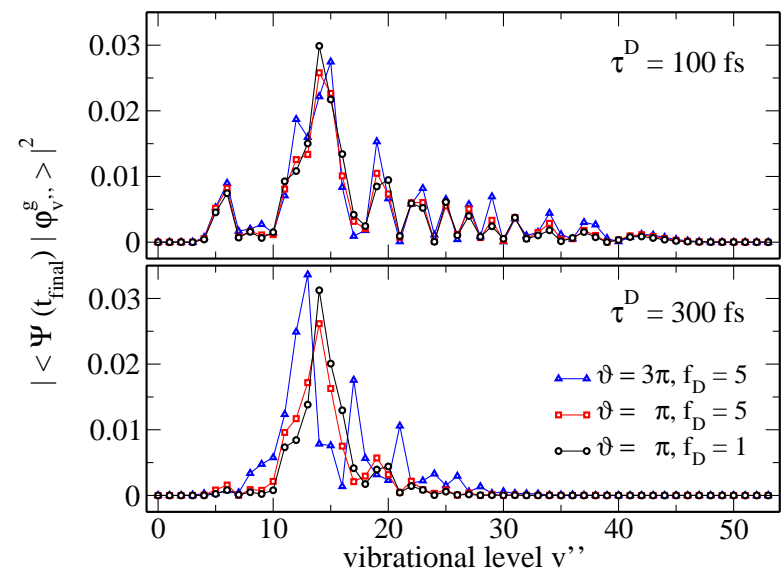

FIG. 9: (Color online) The vibrational distribution of the final ground state wave packet after moderately chirped pulses $\left(f_{D}=5\right.$, cf. Fig. (7) with $\tau^{D}=100 \mathrm{fs}$ (top) and $\tau^{D}=300 \mathrm{fs}$ (bottom) for two different intensities corresponding to $\theta=\pi$ (red squares) and $\theta=3 \pi$ (blue triangles). The vibrational distribution after an unchirped $\pi$-pulse is shown for comparison (black circles).

circles) for both pulse durations. For higher intensities $(\vartheta=3 \pi$, blue triangles), non-perturbative effects start to play a role, and the vibrational distribution is changed: For example, the maximum is not anymore observed for the level with the largest Franck-Condon factor $\left(v^{\prime \prime}=\right.$ 14). Furthermore for $\tau^{D}=300 \mathrm{fs}$, the approximation of an effective two-level system starts to break down due to the long pulse duration. While at higher intensity the dynamics becomes harder to interpret, the overall probability to form bound molecules is increased from $18 \%(\vartheta=\pi)$ to $21 \%(\vartheta=3 \pi)$ for $\tau^{D}=100 \mathrm{fs}$ and from $11 \%$ to $16 \%$ for $\tau^{D}=300 \mathrm{fs}$.

\section{Dependence on the time spent on the excited state}

After excitation by the photoassociation pulse at long range, the wave packet is accelerated toward shorter distances until it reaches the inner barrier of the external potential well (solid blue line in Fig. 10 top panel). While a small part of the wave packet tunnels into the inner potential well, most of it is reflected at the barrier and moves back toward larger distances (dashed red line). The Franck-Condon factors reflect this timedependence of the excited state wave packet (Fig.10 bottom panel, see also Fig. 3). Short and sufficiently intense pulses $\left(\tau^{D}=100 \mathrm{fs}, \vartheta=\pi\right)$ allow for inducing maximum population inversion in both cases, and the final ground state vibrational distributions completely mimic the Franck-Condon factors (data not shown). However, only the wave packet at shorter distances has good Franck-Condon overlap with bound ground state levels, and ground state molecules are formed by the stabilization pulse. For the excited state wave packet at later 


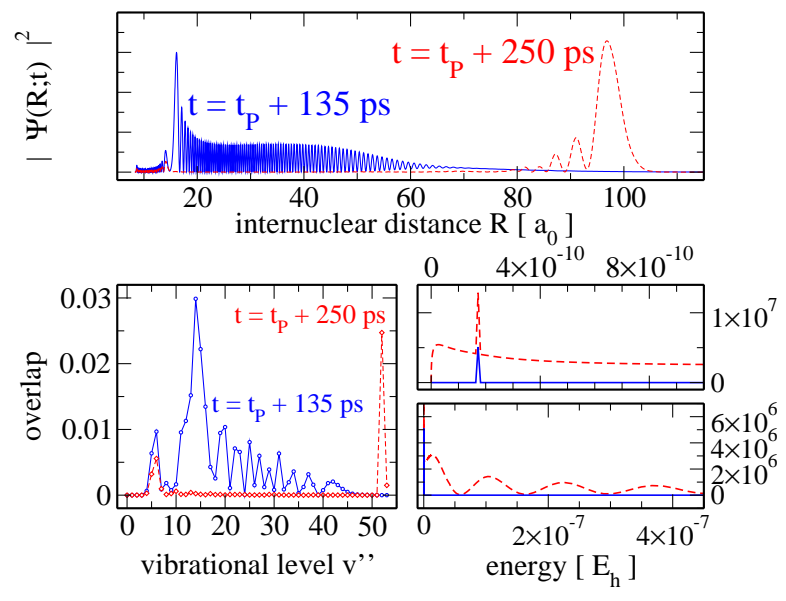

FIG. 10: (Color online) top: The excited state wave packet for two different time delays between pump and dump pulses: $t=$ $t_{P}+T_{\mathrm{vib}} / 2$ (solid blue line) and $t=t_{P}+T_{\mathrm{vib}}$ (dashed red line) ( $t_{P}$ denotes the time where the pump pulse has its maximum). bottom: The Franck-Condon overlap of the excited state wave packets with the ground state bound (left) and the continuum (right) levels.

times, only the last two bound levels of the ground state are populated (except for tunneling), and a considerable amount of population is transferred to the continuum, i.e. two free atoms are created. Fig. 10] confirms our initial assumption that stabilization to ground state molecules is optimal when the excited state wave packet is close to the inner turning point of the outer potential well.

\section{E. Dependence on the sign of the chirp of the photoassociation pulse}

Excited state wave packets after pump pulses with positive $\left(\mathcal{P}_{+}\right.$, red dashed lines $)$and negative chirp $\left(\mathcal{P}_{-}\right.$, blue solid lines) are compared in Fig. 11] at the time of maximum focussing at the inner barrier. The sign of the chirp determines how well the excited state wave packet can be focussed (cf. top panel of Fig. 111). For positive chirp, the wave packet is less focussed, and the maximum probability to form bound molecules is $6 \%$ compared to $21 \%$ for negative chirp. Furthermore, almost half of these $6 \%$ are due to the specific topology of the $0_{g}^{-}$-potential of $\mathrm{Cs}_{2}$, i.e. tunneling to the inner well. Focussing as obtained with $\mathcal{P}_{-}$on the other hand represents a general scheme. As in the previous sections the vibrational distributions after the dump pulse (data not shown) completely mimic the Franck-Condon overlap factors for pulses which induce maximum population transfer (e.g. $\tau^{D}=100 \mathrm{fs}$, $\vartheta=\pi)$. The population of bound levels is then $4.9 \%$ for $\mathcal{P}_{+}$to $18 \%$ for $\mathcal{P}_{-}$.

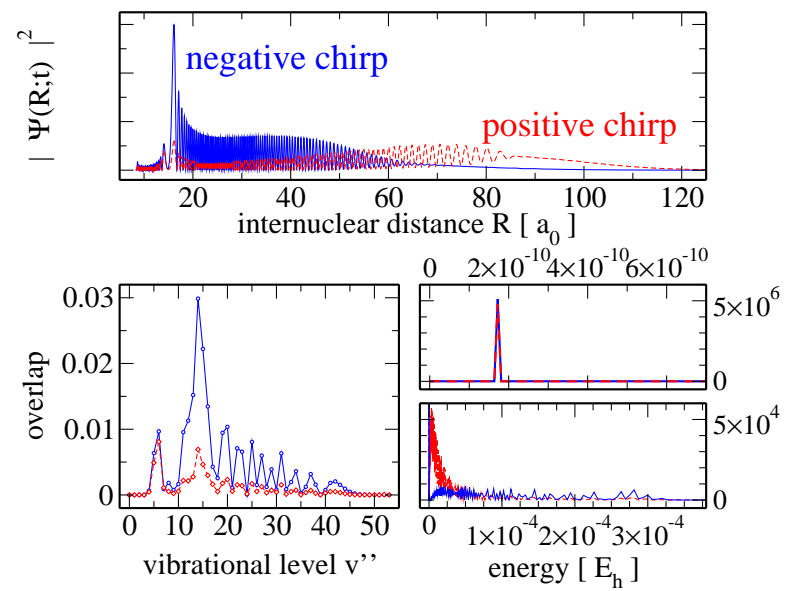

FIG. 11: (Color online) top: The excited state wave packet at $t=t_{P}+135 \mathrm{ps}$ for positive and negative sign of the pump pulse chirp $\left(\mathcal{P}_{-}\right.$and $\left.\mathcal{P}_{+}\right)$. (bottom) The Franck-Condon overlap of the excited state wave packet with the ground state bound (left) and the continuum (right) levels.

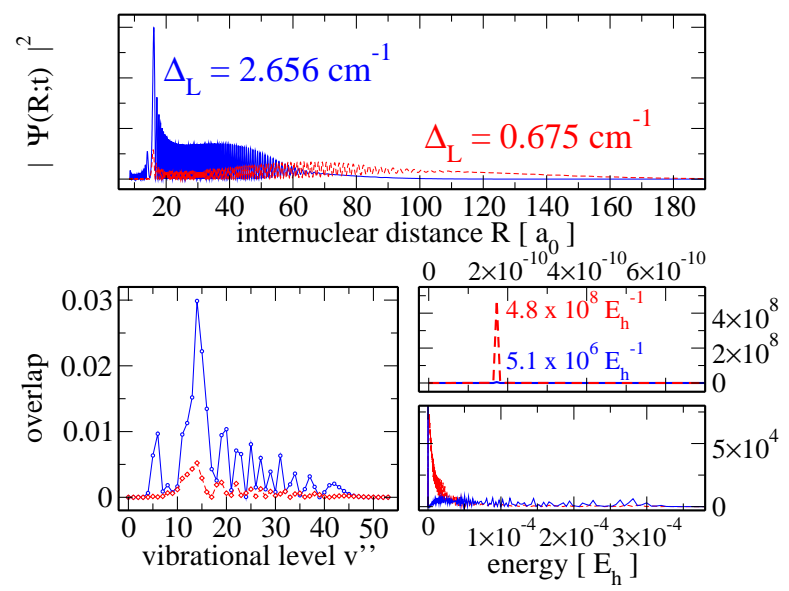

FIG. 12: (Color online) top: The excited state wave packet at $t=t_{P}+T_{\mathrm{vib}} / 2$ for two different detunings of the pump pulse. bottom: The Franck-Condon overlap of the excited state wave packet with the ground state bound (left) and the continuum (right) levels.

\section{F. Dependence on the detuning of the photoassociation pulse from the atomic line}

Fig. 12 compares the excited state wave packet for two different detunings of the pump pulse $\left(\mathcal{P}_{-}\right.$and $\mathcal{P}_{-}^{122}$, respectively). For smaller detuning $\left(\mathcal{P}_{-}^{122}\right)$, higher vibrational levels of the excited state potential get populated. Due to the larger spread of vibrational periods, it is harder to fulfil the condition, Eq. (3), and the wave packet is less focussed (cf. top panel Fig. 12). Furthermore, no tunneling to the inner well of the $0_{g}^{-}$ state occurs for smaller detuning. Hence, the peak in the Franck-Condon overlap factors around $v^{\prime \prime}=6$ is missing. Pulses which achieve maximum population inversion to 


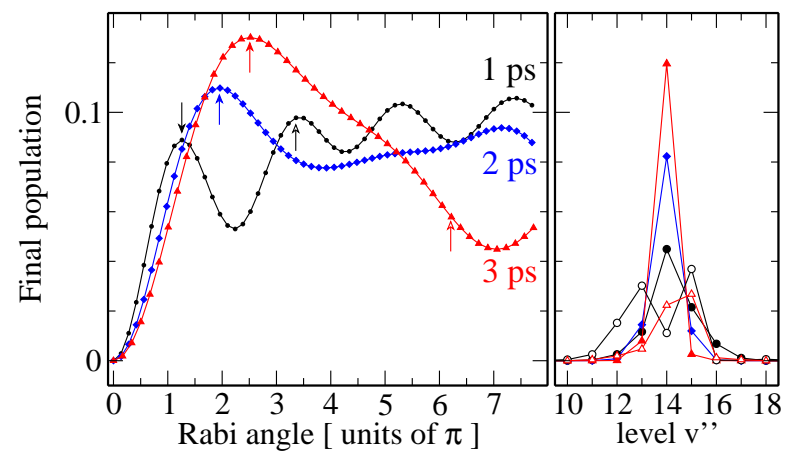

FIG. 13: (Color online) left: Overall ground state population after transform-limited pulses with $\tau^{D}=1$ ps (black circles, 2 ps (blue diamonds), and 3 ps (red triangles) as a function of the Rabi angle. right: Vibrational distributions of the final ground state wave packet corresponding to the points indicated by arrows in the left panel.

the ground state can still be found (e.g. $\tau^{D}=100 \mathrm{fs}$, $\vartheta=\pi$ ). However, the probability of populating bound ground state levels is at $3.4 \%$ considerably smaller. The efficiency of the complete two-color scheme is of course given by the efficiencies of pump and dump steps. One has to keep in mind that since the probability of the photoassociation step is higher for $\mathcal{P}_{-}^{122}$ (see Table I), the two effects compensate and the final number of bound molecules is the same.

\section{HOW TO CREATE GROUND STATE MOLECULES IN A SINGLE LEVEL}

In order to achieve selective population of the level $v^{\prime \prime}=14$, we consider longer pulses, with parameters reported in Table IV As explained in Sec. IIIB pulses with corresponding transform-limited FWHM $\tau^{D}>2$ ps are expected to populate a single vibrational level. Fig. 13 reports the final ground state population after dump pulses with $\tau^{D}=1 \mathrm{ps}$ (black circles), $2 \mathrm{ps}$ (blue diamonds) and 3 ps (red triangles) as a function of the Rabi angle, i.e. pulse intensity on the left, and corresponding vibrational distributions on the right. A pulse with $\tau^{D}=3 \mathrm{ps}$ and moderate intensity indeed excites exclusively a single vibrational level (filled red triangles in Fig. [13] right). The population of this level is $P_{v^{\prime \prime}}=14=0.12$ compared to the total population of $P_{g}^{\text {bound }}=0.13$. A broadening of the vibrational distribution is only observed at fairly high intensity $(\vartheta \sim 6 \pi$, empty red triangles). For $\tau^{D}=1 \mathrm{ps}$, broadening of the vibrational distribution sets in already at lower intensities $\left(\vartheta \sim 3 \pi\right.$, empty black circles). For $\tau^{D}=1 \mathrm{ps}$, oscillations of the final population with the Rabi angle still persist, albeit with much lower contrast than for the broad-bandwidth pulses of Sec.IV] For longer pulses, the impulsive approximation completely ceases to be valid, and the behavior cannot anymore be understood (and employed for control) in terms of an effective two-level

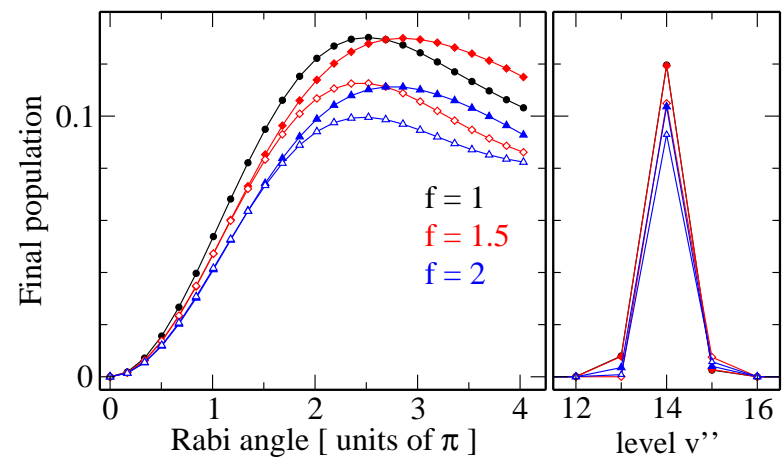

FIG. 14: (Color online) left: Overall ground state population after negatively (filled symbols) and positively (open symbols) chirped pulses with transform-limited FWHM of 3 ps as a function of the Rabi angle. The stretch factor is 1.5 (red diamonds) and 2 (blue triangles), respectively. The results for an unchirped pulse are shown for comparison (black circles). right: Vibrational distributions of the final ground state wave packet corresponding to the maxima of the final ground state population on the left-hand side.

system. Chirping prolongs the pulses even further, and its effect on the final populations and vibrational distributions is investigated in Fig. 14 which compares moderately chirped pulses (stretching factors $f_{D}=1.5$, red diamonds and $f_{D}=2$, blue triangles) to the unchirped case (black circles) for $\tau^{D}=3 \mathrm{ps}$. The final ground state population (blue triangles, $f_{D}=2$ ) is decreased after a chirped pulse as compared to the unchirped case. However, a slight negative chirp (filled red diamdonds, $f_{D}=1.5$ ) does not significantly influence the population transfer. In general, a range of intensities corresponding to Rabi angles $2 \pi \leq \vartheta \leq 3 \pi$ exists where $10 \%$ or more of the excited state wave packet are transferred to the ground state, almost exclusively to the vibrational level $v^{\prime \prime}=14$ (cf. Fig. 14 right). This result implies that some chirping (which might always occur in an experimental setup) does not perturb stabilization into a single level, i.e. the scheme is expected to be fairly robust.

\section{CONCLUSIONS}

The formation of ultracold molecules is presently a very active field. Previous work has demonstrated the advantage of using chirped laser pulses to create ultracold molecules by photoassociation in an assembly of cold atoms [20, 21]. The molecules are created in an electronically excited state and are short-lived. A stabilization step must therefore be implemented. The present theoretical work has discussed a two-color pumpdump experiment where a second pulse, delayed from the photoassociation (pump) pulse, transfers population to deeply bound levels of the ground state, creating stable molecules.

Following Ref. 20], the pump pulse (with picosecond duration and maximum at time $t_{P}$ ) photoassociates Cs 


\begin{tabular}{|c|c|c|c|c|c|c|}
\hline$\omega_{L}$ & $\hbar \delta \omega$ & $\tau^{D}$ & $f_{D}$ & $\chi_{D}$ & delay $t_{D}-t_{P} \mid$ Fig. \\
\hline $11843.5 \mathrm{~cm}^{-1}$ & $20-7 \mathrm{~cm}^{-1}$ & $1,2,3 \mathrm{ps}$ & 1 & 0 & $135 \mathrm{ps}$ & 13 \\
$11843.5 \mathrm{~cm}^{-1}$ & $7 \mathrm{~cm}^{-1}$ & $3 \mathrm{ps}$ & $1.5,2$ & $1.84,1.2 \mathrm{ps}^{-2}$ & $135 \mathrm{ps}$ & 14 \\
\hline
\end{tabular}

TABLE IV: Parameters for the "long" dump pulses considered in Sec. $\mathbb{\nabla}$ central frequency $\omega_{L}^{D}$, temporal width (FWHME) $\tau^{D}$ streching factor $f_{D}$, linear chirp parameter $\chi_{D}$, delay between the maxima of dump and pump pulses. The corresponding figures are reported in the last column.

atoms populating, via an adiabatic transfer mechanism, about 15 vibrational levels in the external well of the $0_{g}^{-}\left(6 s^{2} S_{1 / 2}+6 p^{2} P_{3 / 2}\right)$ potential curve. A vibrational wave packet is created which is bound by $2.1 \mathrm{~cm}^{-1}$ and localized in the region of the outer turning point. After the pulse, the wave packet moves toward the inner barrier of the potential curve (located at $R_{\text {in }}$ ). The chirp parameter of the pump pulse can be chosen such that after half a vibrational period, at time $t_{P}+T_{\mathrm{vib}} / 2$, this wave packet is focussed at the barrier 20]. Our analysis of the Franck-Condon overlap integrals has shown that the overlap with deeply bound levels $v^{\prime \prime}$ of the ground triplet state is then maximum. A vertical transition toward the level $v^{\prime \prime}=14$, bound by $113 \mathrm{~cm}^{-1}$, is even possible, since the outer turning point of the corresponding wave function coincides with $R_{i n}$. We therefore have discussed how population can efficiently be transferred to the ground triplet state by a short dump pulse which should be in the femto- or picosecond domain and optimally delayed from the pump pulse by $T_{\mathrm{vib}} / 2$.

The validity of this intuitive model has been checked with numerical calculations. We have considered two kinds of pulses: broadband pulses populating many vibrational levels of the ground state, with the aim of maximizing the population transfer, and longer pulses for selective population of a single level, $v^{\prime \prime}=14$.

The first set of calculations has been performed for a time delay of $T_{\mathrm{vib}} / 2$. We have shown that for short pulses and a Rabi angle equal to $\pi$, total population inversion can be obtained, i.e. the vibrational wave packet in the excited state is completely transferred to the ground state. Analysis of the wave packet shows that a maximum of $20 \%$ of this population goes to bound levels. The remaining $80 \%$ correspond to continuum states, i.e. the dump pulse also creates pairs of hot atoms. The vibrational distribution reflects the variation of the Franck Condon overlap. Oscillations in the population transfer as a function of the Rabi angle (i.e. laser intensity for a fixed pulse duration) can be suppressed by chirping the dump pulse. Thus, total population inversion is obtained for a wide range of intensities (corresponding to Rabi angles of $\pi$ to $4 \pi$ in our calculations) with an effective $20 \%$ population transfer to bound levels. Employing a chirped dump pulse therefore yields a robust scheme in which the probability to create stable ground state molecules is $20 \%$, independent of the exact value of laser intensity.

By varying the time-delay between the two pulses, a strong variation in the population transfer which follows the variation of the Franck-Condon factors has been observed. Only at shorter distance, the wave packet in the excited state has good Franck-Condon overlap with bound ground state levels, and ground state molecules are formed by the dump pulse. The highest efficiency is achieved in a narrow time window $\left(T_{\mathrm{vib}} / 2 \pm 2 \mathrm{ps}\right)$ during which the wave packet is maximally focussed at the barrier. For different time-delays, only the last two bound levels of the ground state are populated, and a considerable amount of population is transferred to the continuum, i.e. two free atoms are created. Note that without a second pulse, stabilization would occur via spontaneous emission on a nanosecond timescale. The probability for creating ground state molecules is then given by a time average. Since the wave packet spends more time at large distances than close to the barrier, times with unfavorable Franck-Condon overlaps dominate this average. It is therefore essential to employ a second pulse to fully take advantage of the time-dependent probability to create bound ground state molecules after photoassociation with picosecond pulses.

The calculations have confirmed our intuition that stabilization to ground state molecules is optimal when the excited state wave packet is close to the inner turning point of the outer potential well. The best localization of the wave packet at the inner barrier is brought about by negatively chirping the pump pulse. There is yet another advantage when using a negative chirp of the pump pulse: The photoassociation probability is increased by a factor of 8 as compared to a transform-limited pulse. Furthermore, the probability of creating bound molecules by the dump pulse is enhanced by roughly a factor of 3 for a negatively chirped pump pulse as compared to positively chirped or transform-limited ones. The number of stable molecules formed in a pump-dump sequence can be therefore be increased by 24 when appropriately choosing the pulse parameters.

We have also considered a pump pulse with smaller detuning, for which the photoassociation probability is larger. However, due to a decrease of the Franck-Condon overlap, even for the maximally focussed wave packet, the stabilization step is much less efficient. Therefore, 
the total number of stable molecules is roughly the same as in the previous case.

Finally, we have employed longer dump pulses with a duration of about 3 ps to selectively populate the $v^{\prime \prime}=14$ level in the ground state. Up to $12 \%$ of the photoassociated molecules can then be stabilized into a single level, which is fairly efficient. Note that these molecules are created coherently by the pump-dump sequence.

The full discussion of the definition of the optimal pulse should include the possibility of a repetition scheme, considering further pump and dump pulses. In treating a repetition scheme, we also have to take into account how the pump and dump pulses modify the continuum wave function describing two colliding atoms. This is beyond the scope of the present study.

In conclusion, a two-color pump-dump scheme is rather promising for the creation of stable cold molecules. Due to the choice of the $\mathrm{Cs}_{2} 0_{g}^{-}$potential for the photoassociation step, the present work has analyzed formation of molecules in the $v^{\prime \prime}=14$ level of the ground triplet state, bound by $113 \mathrm{~cm}^{-1}$. Many other schemes, e.g. [15, 33], are possible and should be considered to bring population into the $v^{\prime \prime}=0$ level of the ground state which will open the way to the formation of stable molecular condensates. In this respect, we would like to point out the generality of the two-color pump-dump scheme which only requires efficient photoassociation and stabilization mechanisms involving either long range wells in the excited potentials [34] or resonant coupling [16, 17, 18], but is not restricted to a particular system.

\section{Acknowledgments}

We would like to acknowledge fruitful discussions with Ronnie Kosloff and to thank Anne Crubellier for computing independently overlap integrals with a Numerov method in order to check our numerical results. This work has been supported by the Deutsche Forschungsgemeinschaft (C.P.K.) and the European Union in the frame of the Cold Molecule Research Training Network under contract HPRN-CT-2002-00290. The Fritz Haber Center is supported by the Minerva Gesellschaft für die Forschung GmbH München, Germany. Laboratoire Aimé Cotton belongs to Fédération Lumière Matière (CNRS, Université Paris XI).

\section{APPENDIX A: A NOTE ON THE REPRESENTATION OF CONTINUUM STATES WITH A MAPPED FOURIER GRID}

The Fourier grid employs a finite number of grid points and periodic boundary conditions to represent a quantum wave function. For $V(R) \equiv 0$, this corresponds to the particle in a box problem. The scattering continuum is hence represented by a finite number of discrete box states. The highest momentum which is correctly rep- resented on the grid is given in terms of the (constant) spacing $\Delta R$ between two grid points, $p_{\max }=\pi / \Delta R$.

In order to model long-range interactions which require large grids but not small grid steps, a mapping procedure is introduced. The grid steps in the asymptotic region become very large, correspondingly high momenta are cut off. This alters the representation of continuum states on the grid. When diagonalizing the Hamiltonian, two kinds of continuum eigenfunctions are obtained: true box levels and wave functions with an unphysical shape. The latter will be called "cutoff-states" in the following.

The box levels can be easily identified by the scaling of their eigenenergies, $E_{n} \sim n^{2}$ (except for the first few states just above the dissociation limit where the energies are perturbed by the threshold). The corresponding wave functions are standing waves of the box.

The eigenenergies of the cutoff-states approximately scale with some high power $(\sim 20)$ of the box index, the highest eigenenergy being determined by the largest allowed grid step which is a parameter of the mapping. The corresponding eigenfunctions show very fast oscillations and don't obey the boundary conditions at $R_{\text {min }}$ and $R_{\max }$. It can nevertheless be shown that these cutoffstates constitute an effective representation of the true, physical continuum for energies higher than the energy of the last true box level. To this end, the Franck-Condon overlaps of an excited state wave function with the eigenfunctions of all ground state levels (i.e. bound, box and cutoff-levels) are calculated and compared (i) for different mapping parameters and (ii) to Franck-Condon overlaps obtained by Numerov integration [35].

In the first set of calculations, the largest allowed grid step, $\Delta R_{\max }$, is varied. The Hamiltonian is diagonalized for each $\Delta R_{\max }$ and an increasing number of grid points, $N_{R}$. For each $\Delta R_{\max }$, the number of cutoff-states turns out to be independent of $N_{R}$, i.e. increasing $N_{R}$ leads to a larger number of true box states. In particular, for the smallest $\Delta R_{\max }$ and largest $N_{R}$ used $\left(\Delta R_{\max }=0.33 \mathrm{a}_{0}\right.$, $\left.N_{R}=8191\right)$, a continuum representation is obtained which contains almost no cutoff-states (168 vs. 7969 true box states). The excited state wave function is then interpolated to these grids, and the Franck-Condon overlaps are computed. Note, that the Franck-Condon overlaps for continuum states need to include the correct energy renormalization [21]. The Franck-Condon overlaps turn out to be roughly independent of the grid parameters. We are particularly interested in the sum over the Franck-Condon overlaps which determines the probability of dissociating the excited state molecules into hot, free atoms with a short dump pulse (cf. Sec. IV]) This sum is roughly constant for all grids considered, varying between 0.21 for $\Delta R_{\max }=33 \mathrm{a}_{0}$ and 0.17 for $\Delta R_{\max }=0.33 \mathrm{a}_{0}$. The variation is caused by the interpolation of the excited state wave function to the new grids which is not exact, the approximation getting worse for decreasing $\Delta R_{\max }$.

In a second set of calculations, Franck-Condon overlaps with all ground state levels were calculated for selected 
excited state vibrational levels with both the mapped Fourier grid and the Numerov method. Oscillations like those in Fig. [10] are observed. The resolution of these oscillations in the mapped Fourier grid method depends on the number of grid points, $N_{R}$. Good agreement between the two methods is obtained for $N_{R}=1023$, the value used throughout Secs. IV and V]
[1] J. Doyle, B. Friedrich, R. Krems, and F. Masnou-Seeuws, Eur. Phys. J. D, 31, 149 (2004)

[2] E. Donley, N. Claussen, S. Thompson, and C. Wieman, Nature 417 (2002)

[3] N. R. Claussen, E. A. Donley, S. T. Thompson, and C. E. Wieman, Phys. Rev. Lett. 89, 010401 (2002)

[4] J. Herbig, T. Kraemer, M. Mark, T. Weber, C. Chin, H.-C. Nägerl, and R. Grimm, Science 301, 1510 (2003)

[5] K. Xu, T. Mukaiyama, J. R. Abo-Shaeer, J. K. Chin, D. E. Miller, and W. Ketterle, Phys. Rev. Lett. 91, 210402 (2003)

[6] S. Dürr, T. Volz, A. Marte, and G. Rempe, Phys. Rev. Lett. 92, 020406 (2003)

[7] C. A. Regal, C. Ticknor, J. L. Bohn, and D. S. Jin, Nature 424, 47 (2003)

[8] M. W. Zwierlein, C. A. Stan, C. H. Schunck, S. M. F. Raupach, S. Gupta, Z. Hadzibabic, and W. Ketterle, Phys. Rev. Lett. 91, 250401 (2003)

[9] K. E. Strecker, G. B. Partridge, and R. G. Hulet, Phys. Rev. Lett. 91, 080406 (2003)

[10] J. Cubizolles, T. Bourdel, S. J. J. M. F. Kokkelmans, G. V. Shlyapnikov, and C. Salomon, Phys. Rev. Lett. 91, 240401 (2003)

[11] S. Jochim, M. Bartenstein, A. Altmeyer, G. Hendl, C. Chin, S. Riedl, J. H. Denschlag, and R. Grimm, Science 302, 2101 (2003)

[12] S. Jochim, M. Bartenstein, A. Altmeyer, G. Hendl, C. Chin, J. H. Denschlag, and R. Grimm, Phys. Rev. Lett. 91, 240402 (2003)

[13] M. Greiner, C. A. Regal, and D. S. Jin, Nature 426, 537 (2003)

[14] A. Fioretti, D. Comparat, A. Crubellier, O. Dulieu, F. Masnou-Seeuws, and P. Pillet, Phys. Rev. Lett. 80, 4402 (1998)

[15] J. M. Sage, S. Sainis, T. Bergeman, and D. DeMille. Phys. Rev. Lett., 94, 203001 (2005)

[16] C. M. Dion, C. Drag, O. Dulieu, B. Laburthe Tolra, F. Masnou-Seeuws, and P. Pillet, Phys. Rev. Lett. 86,
$2253(2001)$

[17] O. Dulieu and F. Masnou-Seeuws, J. Opt. Soc. Am. B 20, 1083 (2003)

[18] A. J. Kerman, J. M. Sage, S. Sainis, T. Bergeman, and D. DeMille, Phys. Rev. Lett. 92, 153001 (2004)

[19] J. Vala, O. Dulieu, F. Masnou-Seeuws, P. Pillet, and R. Kosloff, Phys. Rev. A, 63, 013412 (2001).

[20] E. Luc-Koenig, R. Kosloff, F. Masnou-Seeuws, and M. Vatasescu, Phys. Rev. A 70, 033414 (2004)

[21] E. Luc-Koenig, M. Vatasescu, and F. Masnou-Seeuws, Eur. Phys. J. D 31, 239 (2004)

[22] J. Cao, Ch. J. Bardeen and K. R. Wilson, Phys. Rev. Lett. 80, 1406 (1998)

[23] J. Cao, Ch. J. Bardeen and K. Wilson, J. Chem. Phys. 113, 1898 (2000)

[24] M. J. Wright, S. D. Gensemer, J. Vala, R. Kosloff, and P. L. Gould. Phys. Rev. Lett. 95, 063001 (2005)

[25] U. Banin, A. Bartana, S. Ruhman and R. Kosloff, J. Chem. Phys. 101, 8461 (1994)

[26] W. C. Stwalley, J. Chem. Phys. 58, 3867 (1973)

[27] I. Averbukh and M. Shapiro, Phys. Rev. A 47, 5086 (1993)

[28] D. G. Abrashkevich, I. Sh. Averbukh, and M. Shapiro, J. Chem. Phys. 101, 9295 (1994)

[29] J. Ahn, T. C. Weinacht, and P. H. Bucksbaum, Science 287, 463 (2000)

[30] M. Vatasescu and F. Masnou-Seeuws, Eur. Phys. J. D 21, 191 (2002)

[31] J. Vala and R. Kosloff, Optics Express 8, 238 (2001)

[32] K. Willner, O. Dulieu, and F. Masnou-Seeuws, J. Chem. Phys. 120, 548 (2004)

[33] C. P. Koch, J. P. Palao, R. Kosloff, and F. MasnouSeeuws. Phys. Rev. A, 70, 013402 (2004)

[34] F. Masnou-Seeuws and P. Pillet. Adv. in At., Mol. and Opt. Phys. 47, 53 (2001)

[35] Anne Crubellier, Private communication (2005) 